\title{
Intelligent Control of a Fuel Cell Power Plant
}

\author{
Tae-Il Choi* and Kwang Y. Lee** \\ *Department of Electrical Engineering, The Pennsylvania State University, University Park, PA 16802 \\ USA (Tel: 814-863-7810; e-mail: tic1@psu.edu) \\ ** Electrical \& Computer Engineering, Baylor University, One Bear Place \#97356, Waco, TX 96798 \\ USA (Tel: 254-710-4188; e-mail: Kwang_Y_Lee@baylor.edu)
}

\begin{abstract}
Fuel cells will become more attractive to mainstream electricity users as they improve in capability and decrease in cost. Molten Carbonate Fuel Cell (MCFC) stack dynamic model was developed to analyze a spectrum of dynamic responses, and a simplified process flow diagram of a fuel cell power plant is presented. The neural network (NN) computing architectures suggest that they may be good candidates for implementing real-time controllers for complicated, nonlinear dynamic systems. A new concept of intelligent setpoint reference governor (I-SRG) using heuristic algorithm will be developed to find the optimal setpoints based on system constraints and performance objectives.
\end{abstract}

\section{INTRODUCTION}

A fuel cell is similar to a battery in that an electro-chemical reaction is used to create electric current. Since the gas reactants can be fed into the fuel cell and constantly replenished, the unit will never run down like a battery. In a typical fuel cell, gaseous fuels are fed continuously to the anode (negative electrode) and an oxidant (i.e., oxygen from air) is fed continuously to the cathode (positive electrode). Appleby (1989) noted that, in theory, any substance capable of chemical oxidation that can be supplied continuously (as a fluid) can be burned galvanically as fuel at the anode of a fuel cell. The catalytic function of electrodes is more important in lower temperature fuel cells and less so in high temperature fuel cells because ionization reaction rates increase with temperature (EG \& G Technical Services Inc., 2002).

There are four main types of fuel cells currently being developed and/or distributed. They include Phosphoric Acid Fuel Cells (PAFC), Molten Carbonate Fuel Cells (MCFC), Solid Oxide Fuel Cells (SOFC), and Proton Exchange Membrane Fuel Cells (PEMFC). Natural gas (methane) is considered to be the most readily available and cleanest fuel (next to hydrogen) for distributed generation applications, so most research for stationary power systems is focused on converting natural gas into pure hydrogen fuel. This is particularly true for low-temperature fuel cells (PEMFC and PAFC). Here, fuel reformers use a catalytic reaction process to break the methane molecule and then separate hydrogen from carbon based gases. High temperature fuel cells such as the MCFC or the SOFC do not require a reformer since the high operating temperature of the fuel cell allows for the direct conversion of natural gas to hydrogen.

\section{MCFC POWER PLANT}

A model of a Direct MCFC stack is investigated as part of an ongoing study of autonomous plant-wide and distributed generation control of fuel cell power plants. The investigation is based on first principles and includes the dominant chemical reactions and simplifying assumptions used to derive the lumped parameter model. Direct fuel cell (DFC) internally reforms methane-containing fuels into hydrogen, partially in an internal reforming unit (RU) and partially at the cells.

A relatively large-scale demonstration of the MCFC technology and its commercialization potential is California's 2-MW Santa Clara Demonstration Project (SCDP). The SCDP contains $125-\mathrm{kW}$ stacks of fuel cells based on DFC technology developed by Fuel Cell Energy (FCE). A simplified process flow diagram (PFD) of the SCDP is shown in Fig. 1. A dynamic model for the SCDP including balanceof-plant (B.O.P.) has been described in (Lukas et al., 2000). A simulation model of the Direct Reforming MCFC stack was implemented for control system applications to fuel cell power plants using the matlab/simulink software.

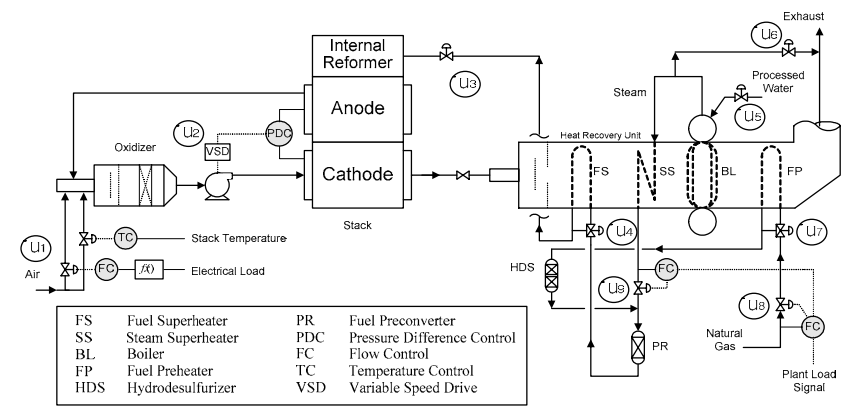

Fig. 1. Simplified process flow diagram for the Santa Clara Demonstration Project

\section{CONTROL SYSTEM FOR THE SCDP}

The operation and control of the SCDP is considered to produce the total stack power. In Fig. 1, 9 control points of the SCDP are displayed as control loops from $\mathrm{u}_{1}$ to $\mathrm{u}_{9}$. First, 
the simulation model is validated at several steady-state operating points, as representatives of the assumed range of operation. Setpoint control laws are then derived for the proper regulation of fuel utilization and steam-carbon ratio. Finally, the plant is subjected to cycling load changes, where all control loops are operational (Lukas et al., 2000; Lukas et al., 2001).

\subsection{The Control Structure of the SCDP}

The control structure of the SCDP power plant is shown in the Fig. 2, where the control system consists of setpoint reference governor (SRG), 9 controllers and the power conditioning system (PCS) for 10 control loops. The PCS converts Plant DC power produced by the stack array to $\mathrm{AC}$ power for grid. The PCS is considered to be an ideal power converter with unity gain and first-order lag dynamics. The Plant DC power output is forced to follow the Plant DC power demand through the 9 controllers and the PCS. The SRG generates the load dependent setpoint values according to Plant DC power demand. These load dependent setpoints are r4, r8, r9, and r10 in Fig. 1. Other setpoints of r1, r2, r3, and $\mathrm{r} 5$ to $\mathrm{r} 7$ are the constant values.

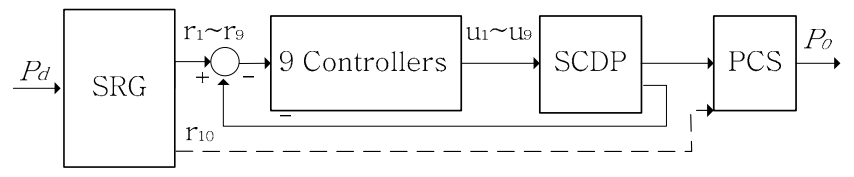

$\mathrm{P}_{\mathrm{d}}$ : AC Power demand, $\mathrm{P}_{\mathrm{o}}:$ AC Power Output

$\mathrm{r}_{1} \sim \mathrm{r}_{10}$ : Setpoint Signals, $\mathrm{u}_{1} \sim \mathrm{u}_{9}$ : Control Signals

Fig. 2. Overall Control Structure of a fuel cell power plant.

Each of the 9 controllers is designed on the basis of a singleloop PI controller capable of responding to the Plant DC power demand. In order to avoid cheap control (large, unrealistic control action) (Kwakernaak and Sivan, 1972), actuator constraints on all loops by limiting the control gains are observed implicitly. Generally, PI controllers are not designed to be model-based because of nonlinear characteristics of the MCFC power plant. Control loop tuning procedures (Shinskey, 1988) are used to stabilize the nominal plant for small perturbations, followed by trial-and-error to expand the operating range.

There are details about the system control that are known only to Distributed Control System (DCS) vendors. For example, it is not known that the setpoint calculations for natural gas flow and steam flow, or whether these were automatically computed by the DCS. RU inlet temperature setpoint is known for several operating points.

Five control loops: stack differential pressure, RU backpressure, steam drum volume, steam drum pressure, and natural gas temperature are all associated with fixed setpoints given by the SRG, invariant with Plant DC power demand. The controllers associated with these control loops are therefore regulators. The SRG determines the four setpoint signals of RU Inlet Temperature, Natural Gas Flow, Steam
Flow, and Stack Temperature depending on Plant DC power demand (Lukas et al., 2002).

The RU inlet temperature setpoint is scheduled with Plant DC power demand. For simplicity, it is assumed that the setpoint is linearly interpolated between the known, optimal setpoints. The operating range covered by the load profile includes the RU temperature setpoints: (1) rated power (1809 $\mathrm{kW}) 1056.5^{\circ} \mathrm{F}$, (2) full power $(2023 \mathrm{~kW}) 1069.2^{\circ} \mathrm{F}$, (3) part load $(1452 \mathrm{~kW}) 1044{ }^{\circ} \mathrm{F}$. Thus, the RU inlet temperature controller is a tracking controller around this time-varying setpoint.

\subsection{Setpoints of Steam and Natural Gas Flows}

In order to determine the proper flows of natural gas and steam from the net Plant DC power demand in the SRG, the fuel utilization is defined as follows:

$U_{f} \equiv \frac{\mathrm{H}_{2, \text { in }}-\mathrm{H}_{2, \text { out }}}{\mathrm{H}_{2, \text { in }}}=\frac{\mathrm{H}_{2, \text { consumed }}}{\mathrm{H}_{2, \text { in }}}$

where $\mathrm{H}_{2, \text { in }}$ and $\mathrm{H}_{2 \text {,out }}$ are the molar flow rates of hydrogen at the fuel cell inlet and outlet, respectively. $\mathrm{H}_{2 \text {,consumed }}$ represents the rate of consumption of hydrogen in the electrochemical reaction. Steam-carbon ratio (s/c ratio) is defined as the molar ratio of steam to methane:

s/c ratio $\equiv \frac{x_{\mathrm{H}_{2} \mathrm{O}}}{x_{\mathrm{CH}_{4}}}=\frac{N_{\mathrm{H}_{2} \mathrm{O}}}{N_{\mathrm{CH}_{4}}}$

Using above relationship, a setpoint for natural gas flow can be determined (Lukas et al., 2001):

$w_{\text {nat gas }}=\frac{4(258) \overline{M_{p r}} I_{\text {sys }}}{2 F(.75)\left(1+2 \mathrm{M}_{\mathrm{H}_{2} \mathrm{O}} / \mathrm{M}_{\mathrm{CH}_{4}}\right)\left(\overline{x_{p r, H_{2}}}+4 \overline{x_{p r, C H_{4}}}+\overline{x_{p r, C O}}\right)}$

where $\mathrm{F}$ is Faraday's constant, $I_{s y s}$ is the total DC current of MCFC, the subscript $p r$ denotes preconverter, and the "bar" denotes nominal value.

\section{INTELLIGENT CONTROL OF A FC POWER PLANT}

The major problem of a complex fuel cell power plant is optimization in operation. To reduce the complexity and provide an optimal operation, an intelligent control will be developed. An intelligent setpoint reference governor (ISRG) using Particle Swarm Optimization (PSO) algorithm will be developed to find the optimal setpoints based on system constraints and performance objectives. This I-SRG will be implemented with neural network to generate the setpoint profile and feedforward control inputs for the Plant DC power demand.

\subsection{Intelligent SRG with Feedforward Controller}

The present system utilizes a nonlinear function which maps the Plant DC power demand to load dependant setpoints of natural gas flow, steam flow, and RU inlet temperature. However, setpoints obtained by a fixed nonlinear function 
cannot provide optimal power plant operation. In other words, the nonlinear mapping function does not provide optimal power plant operation when the Plant DC power demand is changed.

Due to the large stack thermal time constant, there is a significant delay in compensating control in a fuel cell power plant. It is desirable, thus, that PI feedback control is supplemented by additional feedforward control, which can give the advantage that a rapid change in control signal is possible, enabling a much tighter control than with slow feedback alone. The feedforward and feedback control for a fuel cell power plant are shown in Fig. 3, where $P_{d}$ and $P_{o}$ is the Plant DC power demand and the Plant DC power output, respectively.

The I-SRG contains the feedforward controllers, whose outputs are represented as $f_{1} \sim f_{9}$. The output of the SCDP is fed back to the feedback controller, which regulates the output variations due to load disturbances and compensates for the variation in the Plant DC power demand.

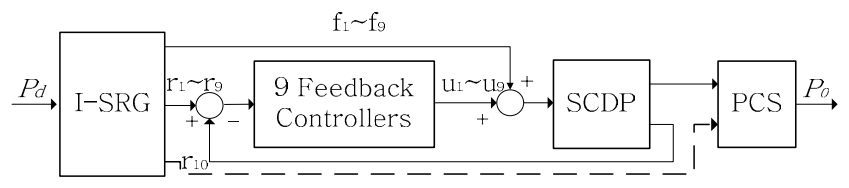

Fig. 3. Overall control structure with I-SRG.

There are various performance objective functions, among which minimization of load-tracking error and fuel consumption are the most important issues to the fuel cell power plant for distributed generation. This multi-objective optimization results in giving the setpoints of the Plant DC power and the RU inlet temperature, which are used to design the feedforward controllers. The multi-objective optimization is performed in objective functions of the I-SRG. By the optimization results, the I-SRG generates not only the load dependent setpoints but also the 9 feedforward control inputs.

Since traditional optimization techniques may often become computationally unattractive or even unacceptable, new optimization techniques need to be applied. Among several modern heuristic optimization techniques, PSO algorithm will be used to solve the objective optimization problem, as it can provide high quality solution with simple implementation and fast convergence (Angeline, 1998).

\subsection{Implementation of I-SRG}

Among 10 setpoints of the SCDP, 4 setpoints are loaddependent. The fuel utilization $U_{f}$ and the steam-carbon ratio are set to $75 \%$ and 2.0 , respectively, for the SCDP as a conservative specification. Then, Steam Flow and Natural Gas Flow can be calculated as (2) and (3), respectively, from a given Plant DC power. Therefore, optimization of setpoints of RU Inlet Temperature and Plant DC power will be considered to solve the multi-objective optimization problem.

The function of the I-SRG is to design optimal mappings from the Plant DC power demand to the setpoints of RU Inlet Temperature and Plant DC power. The I-SRG can be realized by finding all feasible operating points which satisfy all imposed constraints. The I-SRG performs the design process in three steps shown in Fig. 4.

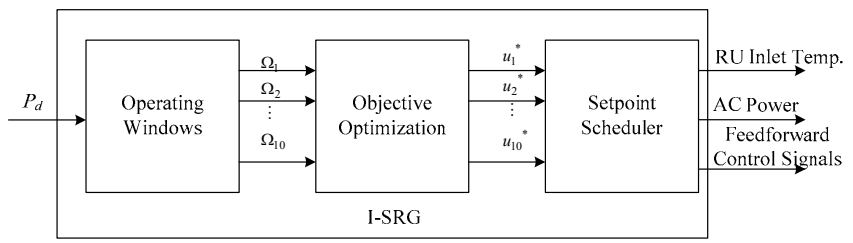

Fig. 4. Configuration of an I-SRG.

- Determination of the feasibility regions $\left(\Omega_{1}, \Omega_{2}, \ldots, \Omega_{10}\right)$ for the decision variables $\vec{u}=\left(u_{1}, u_{2}, \ldots, u_{10}\right)$

- Solution of the multi-objective optimization problem to find optimal steady-state control inputs $\vec{u}^{*}=\left(u_{1}^{*}, u_{2}^{*}, \ldots, u_{10}^{*}\right)$

- Calculation of the RU Inlet Temperature and Plant DC power setpoints through evaluation of the steady-state model of the unit.

The decision variables are candidate steady-state control inputs for control valves, $\vec{u}=\left(u_{1}, u_{2}, \ldots, u_{10}\right)$. To obtain feasibility regions $\left(\Omega_{1}, \Omega_{2}, \ldots, \Omega_{10}\right)$, the value of the Plant DC power demand is changed from $70 \%(1,498 \mathrm{~kW})$ to $100 \%$ $(2,128 \mathrm{~kW})$ by $5 \%(106 \mathrm{~kW})$. The maximum temperature of the stack is limited to $1,250{ }^{\circ} \mathrm{F}$ and the temperature of the rated power is set to $1056.5^{\circ} \mathrm{F}$. Fig. 5 shows the Powercontrol input operating windows, where each one is normalized corresponding to the full power range.
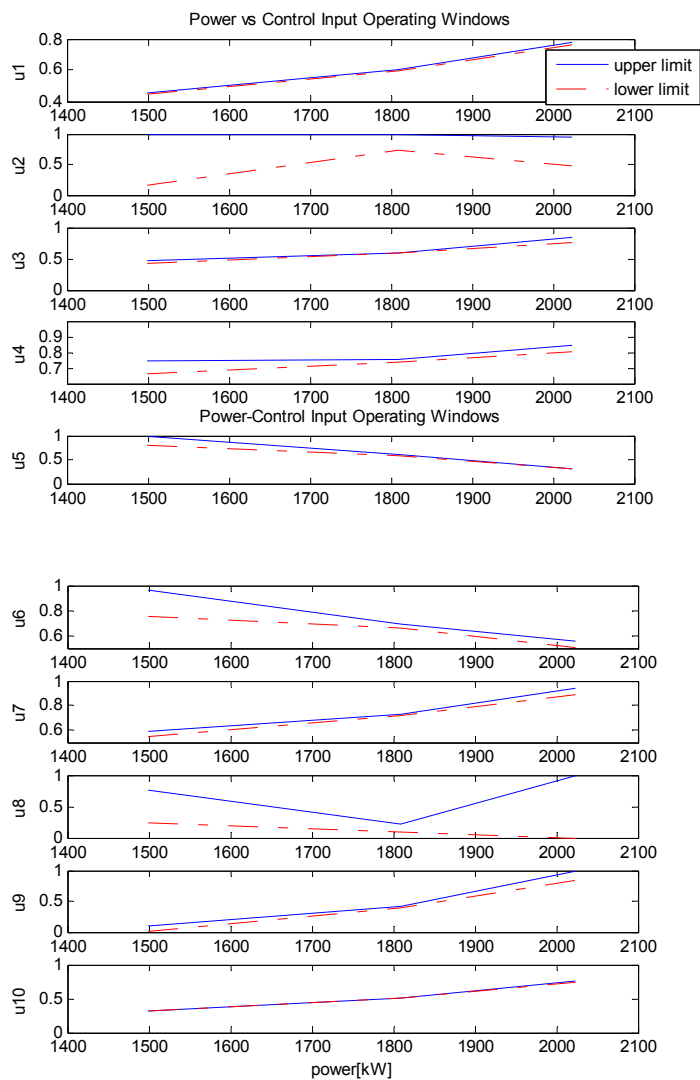

Fig. 5. Power-control input operating windows. 
For the first step, it is necessary to find the steady-state model for calculation of the setpoints by using the obtained feasibility regions $\left(\Omega_{1}, \Omega_{2}, \ldots, \Omega_{10}\right)$. A NN which is known to make the best approximation for nonlinear systems can be used to generate the steady-state models as follows:

Power: $E(\vec{u})=E\left(u_{1}, u_{2}, \ldots, u_{10}\right)$

RU Inlet Temperature: $T(\vec{u})=T\left(u_{1}, u_{2}, \ldots, u_{10}\right)$

In most control applications, the real-time implementation is very important, and thus the neuro-controller also needs to be designed such that it converges with a relatively small number of training cycles. Since this model is a static mapping rather than dynamic response, the network model can be simplified with feedforward NN. In the network, 21 neurons are used for the hidden layer neuron by a rule of thumb and it showed fast convergence within 2,000 iteration number.

Fig. 6 shows the validation of NN setpoint scheduler for Plant DC power and RU Inlet Temperature. Performances represent successful results, showing very small errors between the steady-state output responses and the estimated values of setpoint scheduler.
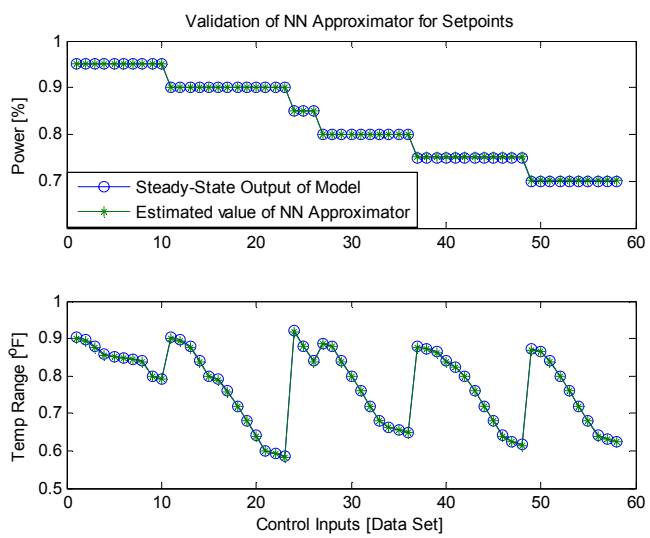

Fig. 6. Validation of NN approximation for setpoint.

For the next step, the optimal solution, $\vec{u}^{*}=\left(u_{1}^{*}, u_{2}^{*}, \ldots, u_{10}^{*}\right)$, is found using the PSO, which is processed to solve the multiobjective optimization for load following and fuel conservation. An objective function for minimization of loadtracking error is formulated as follows:

$J_{1}(\vec{u})=\left|P_{d}-E(\vec{u})\right|$.

The Plant DC power setpoint $E(\vec{u})$ can track to the Plant DC power demand $\left(P_{d}\right)$ as closely as possible by minimizing the objective function $J_{1}(\vec{u})$. With the operating windows and performance objective function (6), the PSO technique will be used to find the optimal inputs $\vec{u}^{*}$.

The plant efficiency can be defined as follows:

$\eta=\frac{P_{o}}{P_{\text {fuel }}}, P_{\text {fuel }}=M_{\mathrm{CH}_{4}} \cdot \mathrm{LHV}_{\mathrm{CH}_{4}} \cdot \mathrm{N}=800 \mathrm{~N}$, where $\mathrm{N}$ is the molar flow of natural gas, $M_{\mathrm{CH}_{4}}=16 \mathrm{~kg} / \mathrm{kmol}$, and Lower Heating Value $\mathrm{LHV}_{\mathrm{CH}_{4}}=50^{3} \mathrm{~kJ} / \mathrm{kg}$. An objective function for the plant efficiency is formulated as follows:

$$
J_{2}(\vec{u})=u_{7}
$$

where $U_{7}$ is the natural gas flow control input. $J_{2}(\vec{u})$ is required to be minimized to increase the plant efficiency.

In the multi-objective optimization, the objective functions are often in conflict with one another when performing the optimization. Thus, it is proposed to minimize the maximum deviation of the objective. The maximum deviation of multiobjective functions is defined as follows:

$\delta_{m}=\max _{i=1,2} \delta_{i}, \delta_{i} \geq 0$,

$\delta_{i}=\beta_{i}\left|J_{i}(\vec{u})-J_{i}(\vec{u})^{*}\right|, \quad i=1,2, \quad \vec{u} \in \Omega$,

$J_{i}(\vec{u})^{*}=\min \left\{J_{i}(\vec{u}) ; \vec{u} \in \Omega\right\}, i=1,2$.

Here $\delta_{m}$ is the maximum deviation of the multi-objective functions, $\delta_{i}$ is the weighted deviation, $\beta_{i}$ is the preference value, $J_{i}(\vec{u})^{*}$ is the minimum possible value of the single objective function $J_{i}$, and $\Omega$ is the solution space.

The PSO algorithm is used to solve the multi-objective optimization problem (10) with a given vector of preference value $\vec{\beta}=\left[\beta_{1}, \beta_{2}\right]$. After finding the optimal solution, $\vec{u}^{*}=\left(u_{1}^{*}, u_{2}^{*}, \ldots, u_{10}^{*}\right)$, the setpoint scheduler is applied to map the optimal solution into Plant DC power $E\left(\vec{u}^{*}\right)$ and RU Inlet Temperature $T\left(\vec{u}^{*}\right)$. Finally, the setpoint scheduler outputs the optimal solution $\left(u_{1}^{*}\right.$ to $\left.u_{9}^{*}\right)$ as the feedforward control input $\left(f_{1}\right.$ to $\left.f_{9}\right)$.

A plant DC power demand profile for the I-SRG operation is given in Fig. 7. The plant DC power demand profile has different rising and falling slopes and levels of constant powers. By using the power-control input operating windows of Fig. 5, the solution spaces $\left(\Omega_{1}, \Omega_{2}, \ldots, \Omega_{10}\right)$ can be generated with respect to the plant DC power demand profile. Fig. 8 shows the generated solution spaces $\left(\Omega_{1}, \Omega_{2}, \ldots, \Omega_{10}\right)$.

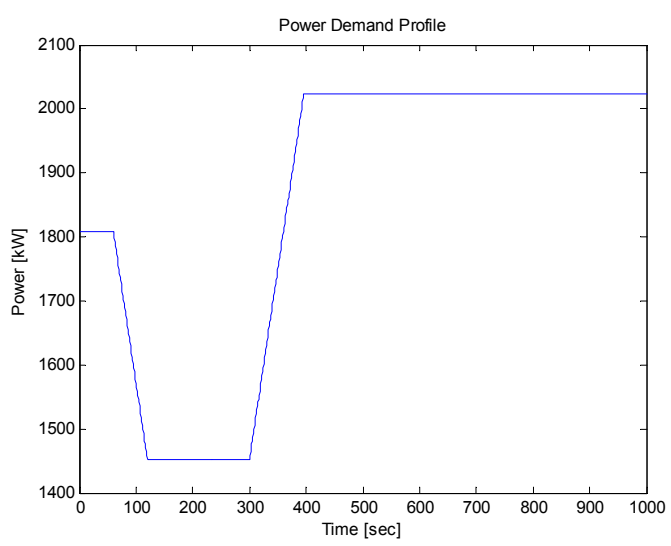

Fig. 7. The plant DC power demand profile. 

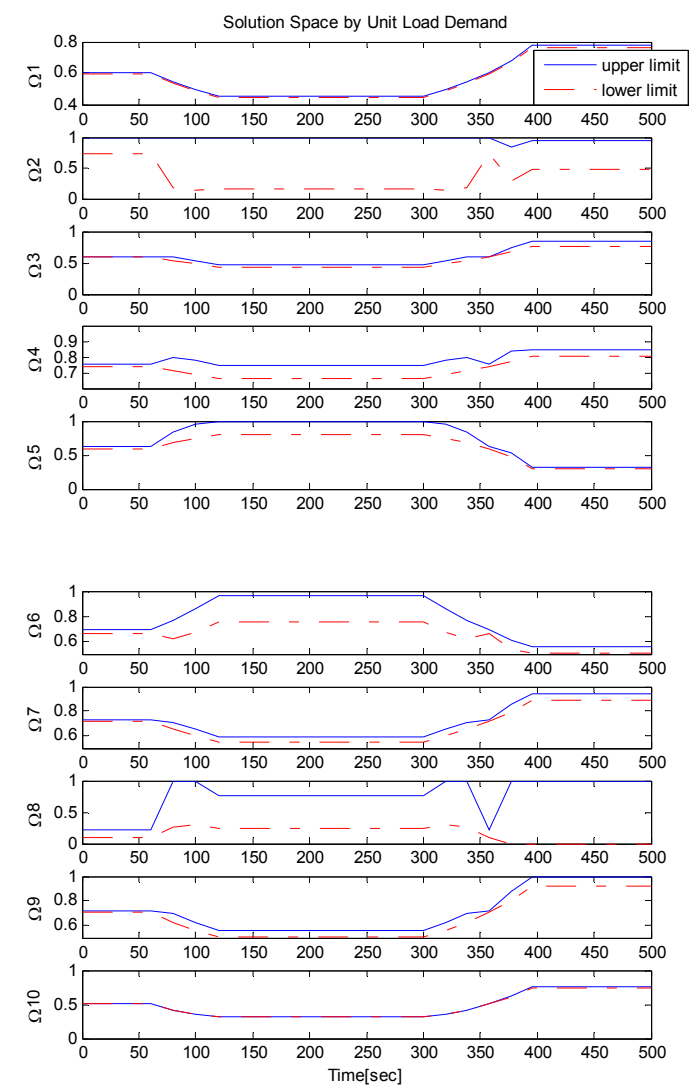

Fig. 8. Solution space by the given plant DC power demand.

\section{SIMULATION RESULTS}

The I-SRG is operated on the given Plant DC power demand profile with two different cases. The Case 1 means $\vec{\beta}=[1,0]$, and in the Case $2, \vec{\beta}$ is set to $[1,0.001]$ to put more weights on load tracking than plant efficiency objective. The PSO is processed for the multi-objective optimization with predefined objective functions and the preference values.

Case 1: minimize $J_{1}(\vec{u})$

Case 2: minimize $J_{1}(\vec{u})$ and $J_{2}(\vec{u})$.

The plant DC power and RU Inlet Temperature setpoints are obtained by the I-SRG and they are shown in Figs. 9 and 10. The Plant DC power setpoint $E\left(\vec{u}^{*}\right)$ are almost the same for both cases as the Plant DC power demand in Fig. 9. However, the conflicting requirements cause slight difference between the Plant DC power setpoint and the Plant DC power demand. The RU Inlet Temperature setpoint $T\left(\vec{u}^{*}\right)$ in Fig. 10 is mapped for different number of objective functions. Different RU temperatures can produce the same amount of power. All the plant DC power setpoint results show that the I-SRG can perform well in the multi-objective optimization problem since the temperature setpoints need to be adopted only when the unit load demand is changed during the load cycle.

The SCDP power plant is simulated with the obtained setpoints $E\left(\vec{u}^{*}\right), T\left(\vec{u}^{*}\right)$ and feedforward control inputs $f_{l}$ to $f_{9}$. Fig. 11 shows the plant DC power demand and the output, which proves satisfactory results for load tracking. RU Inlet Temperature outputs are shown in Fig. 12. When the RU temperature setpoints are varied in Case 1, stack temperature outputs were close to the conventional ones. However, when two setpoints with additional fuel utilization setpoint $\left(\mathrm{U}_{\mathrm{f}}\right)$ are varied, stack temperature showed some fluctuations being different from conventional ones.

As shown in Fig, 13, the plant efficiency of the fuel cell power plant can be improved by using I-SRG. When two setpoints (RU Inlet temperature Td and fuel utilization Uf) are varied to use I-SRG, the plant efficiency was improved significantly. However, some control inputs showed abnormal values, which were caused by constraints of this mathematical simulation model. Therefore, only RU temperature setpoints can be varied to improve the plant efficiency in this simulation model. In real power plant, however, if the control inputs show admissible values, the setpoint of fuel utilization could be varied to improve the plant efficiency to expected levels.

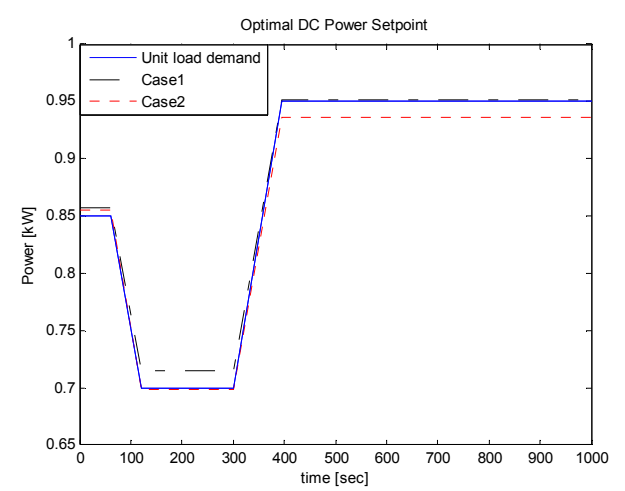

Fig. 9. Plant DC power setpoint trajectories (70-100\%).

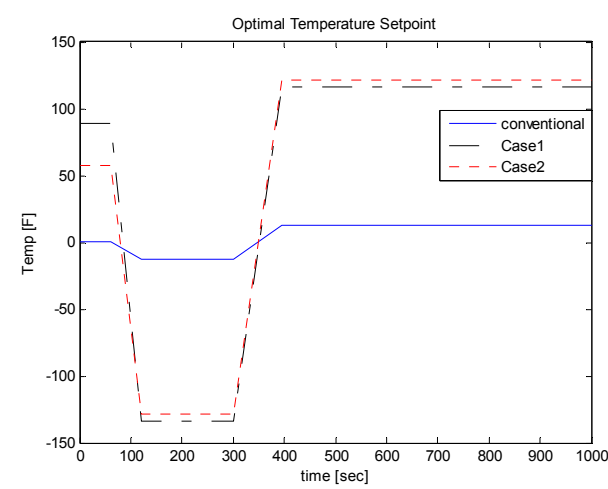

Fig. 10. RU Inlet temperature setpoint trajectories. 


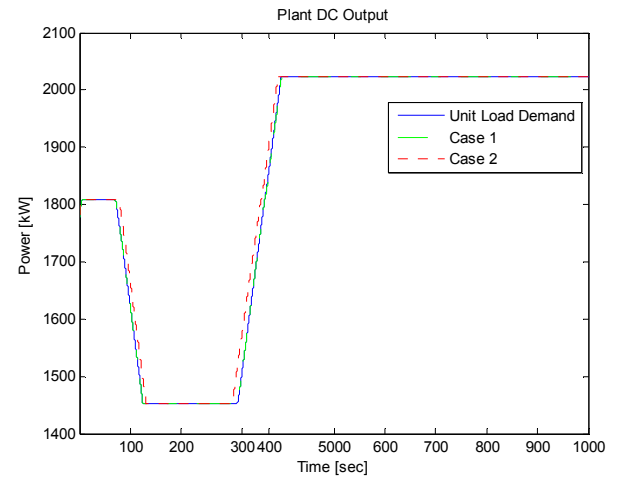

Fig. 11. Plant DC power outputs of simulation results.

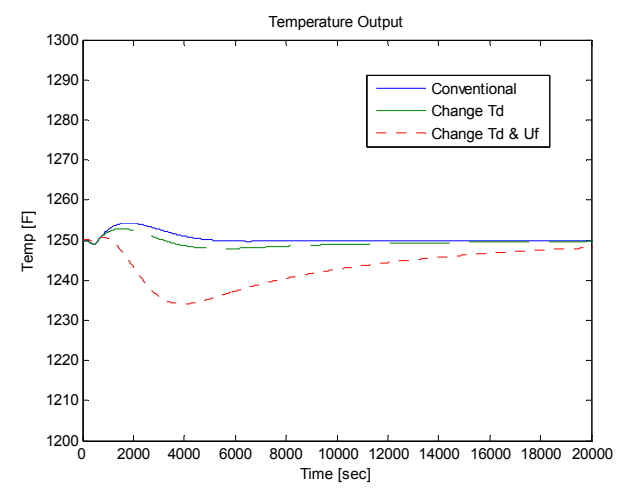

Fig. 12. RU Inlet temperature outputs of simulation results.

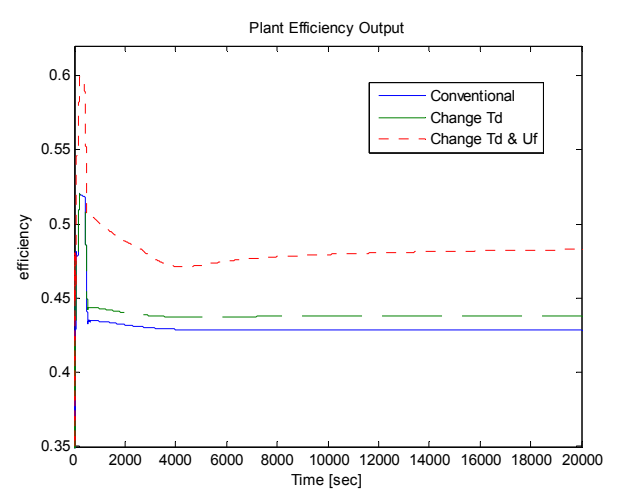

Fig. 13. Comparison of fuel cell efficiency.

\section{CONCLUSIONS}

The major problems in power plant operation including fuel cells are the coupling problems among systems and optimization problems. A new concept of intelligent controller, intelligent setpoint reference governor (I-SRG), using heuristic algorithm and neural network is developed for a fuel cell power plant. Among several objective functions, load tracking and plant efficiency are solved to find optimal setpoints. Simulation results proved that load changes were followed successfully and the plant efficiency was improved using the I-SRG. The intelligent control techniques could be useful for other power plants according to their objectives.

\section{REFERENCES}

Angeline, P. (1998). Using selection to improve particle swarm optimization. Proceeding of IEEE International Conference on Evolutionary Computation, pp. 84-89.

Appleby, A. J. and F. R. Foulkes. (1989). Fuel Cell Handbook, Van Nostrand Reinhold, New York, NY.

EG \& G Technical Services, Inc. (2002). Fuel Cell Handbook, 6th edition, U.S. Department of Energy Office of Fossil Energy, Morganton, West Virginia, Nov.

Farooque, M. (1991). Development of Internal Reforming Carbonate Fuel Cell Technology, Final Report. pp. 3-6 to 3-11, prepared for U.S. DOE/METC, DOE/MC/23274-2941, Oct.

Hirschenhofer, J. H., D. B. Stauffer, , R. R. Engleman, , and M. G. Klett, (1998). Fuel Cell Handbook. U.S. Dept. of Energy,.

Kwakernaak, H. and R. Sivan. (1972). Linear Optimal Control Systems, Wiley, New York.

Lukas, M. D., K. Y. Lee, and H. Ghezel-Ayagh> (2000). Operation and Control of Direct Reforming Fuel Cell Power Plant. Proc. of IEEE Power Engineering Society $W M$, Jan.

Lukas, M. D., K. Y. Lee, and H. Ghezel-Ayagh. (2000). Modeling, Simulation, and Control of Direct Reforming Molten Carbonate Fuel Cell Power Plant. The Fourth IFAC Symposium on Power Plants \& Power Systems Control, pp. 127-134, Apr. 26-29.

Lukas, M. D., K. Y. Lee, and H. Ghezel-Ayagh (2001). Performance Implications of Rapid Load Changes in Carbonate Fuel Cell Systems. Proc. of IEEE Power Engineering Society Winter Meeting, \#2001WM255, Jan. 2001.

Lukas, M. D., K. Y. Lee, and H. Ghezel-Ayagh (2002). Modeling and Cycling Control of Carbonate Fuel Cell Power Plants, Control Engineering Practice, Vol. 10, pp. 197-206, 2002.

Shinoki, T., M. Matsumura, and A. Sasaki. (1995). Development of an Internal Reforming Molten Carbonate Fuel Cell Stack. IEEE Trans. on Energy Conversion, Vol. 10, No. 4, Dec.,.

Shinskey (1988). F. G., Process Control Systems, Application, Design, and Tuning, Mc-Graw Hill, New York. 